\title{
Transfer from recency learning to corresponding two-category classification learning
}

\author{
BENTON J. UNDERWOOD and ROBERT A. MALMI \\ Northwestern University, Evanston, Illinois 60201
}

\begin{abstract}
This experiment tested the hypothesis that recency judgments, used to measure temporal coding, were a special case of two-category classification (TCC) learning. Following four recency-learning trials on a 60-word list, the subjects learned to classify each item as an underlined item or as a nonunderlined item. The underlined items had been the correct items on the recency trials, the nonunderlined items had been incorrect. A small amount of negative transfer was found. Although the performances on unrelated recency-learning tasks and on TCC tasks were substantially correlated, there was no consistency in the correlations when the two tasks were related as described above. It was concluded that the TCC task cannot be used as a model for recency learning. Word frequency was manipulated as a secondary variable; neither TCC learning nor recency learning was influenced by it.
\end{abstract}

Recency judgments have been used to index the acquisition of temporal codes for words within a list. In using this technique, the investigator presents a series of words (or other stimuli) one at a time for study. On the test trial, pairs of words from the list are shown to the subjects and they must choose the word in each pair that occurred most recently in the study order. Correct judgments increase steadily over trials.

It has been proposed that recency judgments are mediated primarily by a type of two-category (TCC) classification learning (Underwood, 1977). The two words within a test pair for recency judgments are said to have been classified in a way that would produce temporal information. An item might be classified as falling into the first half of the list or into the second half of the list. Or, an item might be classified as having occurred in the early part of the list or the later part. For some pairs, the two categories may be first or second. There may be three categories, such as early, middle, or late, but whatever the categories to which items are assigned, at least one word in a test pair is said to be learned as belonging to a category that carries direct temporal information. The theory simply asserts that the understanding of how within-lists recency judgments are acquired will occur when an understanding of TCC learning is acquired.

One of the obvious implications of the theory is that there should be an appreciable correlation between TCC learning and recency learning. To find a strong correlation in a case like this cannot be used to support the theory, but such a relationship clearly recommends

This research was supported by the Personnel and Training Research Programs, Psychological Sciences Division, Office of Naval Research, under Contract N00014-76-C-0270, Contract Authority Identification NR 154-371. Reproduction in whole or in part is permitted for any purpose of the United States government. further work using more direct theoretical tests (Underwood, 1975). Without giving details, it may be reported that in unpublished work, a correlation of .69 was found between the scores on the two types of tasks for 30 subjects. As will be seen, comparable values were found in the present study.

In the experiment to be reported here, the subjects first learned to make recency judgments. On a transfer test, the recency lists were changed into TCC lists in which the correct items on the recency test became the underlined items on the TCC task and the incorrect items became the nonunderlined items on the TCC task. If the theory is appropriate, the transfer between the two tasks should be heavy and positive. As a secondary matter, word frequency was varied to determine its influence on recency judgments and on TCC learning.

\section{METHOD}

Lists

There were four frequency levels, each represented by 60 words. All words had four letters, all were nouns, and most named concrete objects. The four frequency levels were defined by Thorndike and Lorge (1944) as follows: FL-1, frequencies of 1-5 (G count); FL-2, frequencies of 10-15; FL-3, frequencies of $20-49 ; \mathrm{FL}-4$, all words were AA.

The words were placed randomly in the lists, and the pairs were chosen for the recency tests so that three lag levels were defined, with 10 pairs representing each lag. For each test pair, lag was defined as the number of words falling (on the study list) between the two words. The lag levels were short $(2,3$, or 4 intervening words), medium $(10,11)$, or long (30). All 60 words in the lists were tested.

The TCC lists were constructed from the same words used to form FL-1 and FL-4. Thus, the TCC tasks were represented only by the two extremes of frequency. For these TCC lists, the 30 words that were correct in the recency pairings were underlined on the study trials; those that were incorrect were not underlined. 


\section{Procedure and Subjects}

There were four groups of 20 subjects each, assigned to conditions by a block-randomized schedule. The initial conditions consisted of the recency learning as a function of four frequency levels, one group being assigned to each level. There were four study-test cycles, with the words being presented at a 3-sec rate on the study trials. The tests for recency discrimination were unpaced. The order of the pairs on the test sheets differed for each of the four test trials.

We will identify each group in terms of the frequency level of the first list (recency-judgment list). Groups FL-1 and FL-2 were both given the TCC task using the FL-1 words used for recency judgments; Groups FL-3 and FL-4 were both given the TCC task using the FL -4 words. Thus, Groups FL-1 and FL-4 should show positive transfer to the classification tasks from the recency lists if recency discrimination is a case of TCC learning. Group FL-2 served as a control baseline for Group FL-1 on the TCC task, and Group FL-3 served as the control for Group FL-4. Finally, a direct comparison between Groups FL-3 and FL-2 on the TCC task would tell if extreme differences in word frequency influenced learning.

All subjects were given two study-test cycles on the TCC lists. The study trials were conducted at a 3 -sec rate and the test trials were paced, using the same rate. The subjects responded "yes" or "no" to each item to indicate whether it had been underlined on the test trials. It seemed necessary to use the paced test to prevent the subjects from reconstructing the recency list and then deriving classification information from the reconstructed list. The subjects were fully informed concerning the nature of the TCC test prior to the first study trial and again before the first test trial. The subjects in Groups FL-1 and FL-4 were not told that the underlined words in the TCC task were the correct words for the recency pairs. A different order of the words was used on each of the four trials (two study and two test) for the TCC task.

\section{RESULTS AND DISCUSSION}

\section{Recency Judgments}

Word frequency did not have a reliable influence on the recency judgments. The mean correct recency judgments per trial (with standard deviations) for Groups FL-1, FL-2, FL-3, and FL-4 were 6.98 (.75), 6.89 (.81), $6.90(.99)$, and 6.27 (1.06), respectively. Because the means were based on 10 recency tests, the values can be changed to percentages by moving the decimal point one place to the right. Both lag $(\mathrm{F}=75.82)$ and trials $(\mathrm{F}=35.51)$ produced reliable effects. Across the four lists and across the four trials, the mean numbers of correct recency judgments were 24.00 (4.36), 26.76 (5.01), and 30.31 (4.71), for short, medium, and long lags, respectively.

\section{Two-Category Classification}

It will be remembered that on the TCC task, Group FL-2 served as the control for Group FL-1, and Group FL-3 served as the control for Group FL-4. The mean pertormance measures are shown in Figure 1. There were 60 items on the classification task; the scores ranged roughly from $67 \%$ correct to $88 \%$ correct. A correct response was identified as responding "yes" when a word had been underlined and "no" when it had not been underlined on the study trial. The

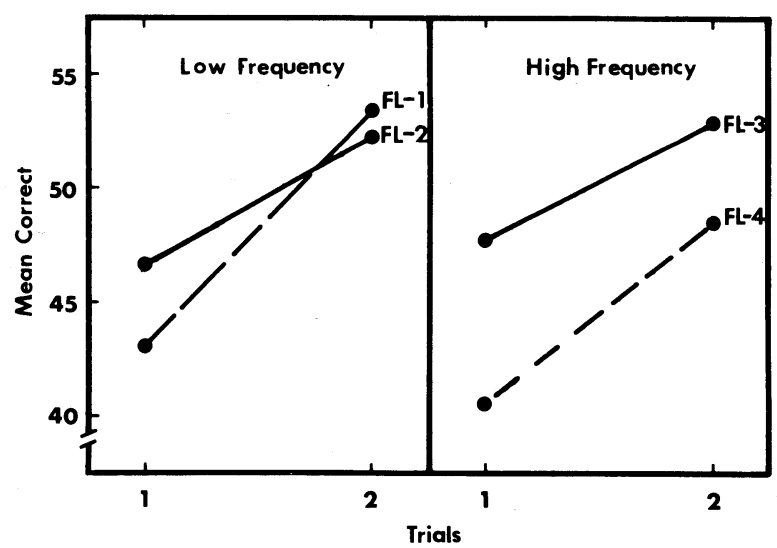

Figure 1. Transfer from recency judgments to two-category classification learning of the same words as related to frequency level and classification-learning trial.

surprising fact shown by these data is that the transfer was negative; performance on the TCC task was retarded by having it made up of words that had previously appeared in the recency-discrimination task. This was true in spite of the fact that all underlined words on the TCC task had been correct words for the recency discriminations. The negative transfer effect was reliable $[F(1,76)=5.72, p<.02]$, and although the amount of negative transfer appears less for the low-frequency words than for the high-frequency words, the interaction was not reliable $[\mathrm{F}(1,76)=2.08, \mathrm{p}>.05]$. In any absolute sense, the negative transfer was not great, but that no positive transfer was observed is obviously very troublesome for the theory that presumes that recency discriminations represent a form of TCC learning.

One other fact given in Figure 1 must be mentioned. The difference between Groups FL-2 and FL-3 was small and nonsignificant statistically. The TCC task given Group FL-2 was made up of low-frequency words; the task given Group FL-3 was made up of high-frequency words. Thus, word frequency does not influence TCC learning.

\section{Correlations}

The theory that recency discrimination fits the paradigm of the TCC task leads to the expectation that the correlation between recency scores and TCC scores should be very high. The correlation for Group FL-1 was .29 , that for Group FL- $4, .55$. The theory could probably tolerate the second correlation, but certainly not the first. It was earlier reported that the skills involved in learning recency judgments were rather highly correlated with the skills invelved in TCC learning. Groups FL-2 and FL-3 provide further tests of this relationship. For FL-2, the correlation was .63, for FL-3, .51.

Two findings seriously question the idea that the learning of recency discriminations is to be understood 
by studying the learning underlying the TCC task. The first is the failure to show positive transfer from recency learning to two-category learning. Even if several different categories were used to classify items in learning recency discriminations, it seems that the category label representing the most recent item in a pair could be translated readily into the appropriate category (underlining) on the TCC task. Strangely enough, the fact that there was negative transfer might be viewed as being more favorable toward the theory than would be true had there been no transfer of any kind. The negative transfer indicates that the two tasks made contact in some way; the associations involved in both tasks had some overlap. Nevertheless, the nature of the overlap is not as expected.

The second finding that is difficult for the theory is the relatively low correlation between the scores for the two types of learning for Group FL-1. It must necessarily follow from the theory that a subject who learns recency discriminations rapidly will also learn the TCC task based on the same items rapidly, and this was not found.

\section{REFERENCES}

Thorndike, E. L., \& LoRgE, I. The teacher's wordbook of 30,000 words. New York: Bureau of Publications, Teachers College, 1944.

UNDERWOOD, B. J. Individual differences as a crucible of theory construction. American Psychologist, 1975, 30, 128-134.

UNDERWOOD, B. J. Temporal codes for memories: Issues and problems. Hillsdale, N.J: Lawrence Erlbaum, 1977.

(Received for publication December 27, 1977.) 